\title{
Neonatal Determinants of Mothers' Affective Involvement in Newly Delivered Cameroonian Women
}

\author{
Georges Pius Kamsu Moyo*, Raïssa Monayong Mendomo, Sonia Zebaze, Laura Kuate Makowa, \\ Christiale Batibonack, Audrey Thérese Mbang
}

Department of Pediatrics, Faculty of Medicine and Biomedical Sciences, University of Yaoundé I, Yaoundé, Cameroon

Email address:

kamsuzicfried@yahoo.fr (G. P. K. Moyo)

${ }^{*}$ Corresponding author

\section{To cite this article:}

Georges Pius Kamsu Moyo, Raïssa Monayong Mendomo, Sonia Zebaze, Laura Kuate Makowa, Christiale Batibonack, Audrey Thérese Mbang. Neonatal Determinants of Mothers' Affective Involvement in Newly Delivered Cameroonian Women. Journal of Family Medicine and Health Care. Vol. 6, No. 2, 2020, pp. 125-128. doi: 10.11648/j.ajp.20200602.21

Received: February 7, 2020; Accepted: February 25, 2020; Published: March 17, 2020

\begin{abstract}
Background: Emotional instability represents a common psychological impairment experienced by newly delivered women, susceptible to impact mothers' affective involvement and hence mother-infant bonding. It may occur as early as during the first two weeks of early postpartum, manifesting firstly with the baby blues also known as postpartum or maternity blues, and secondly with postpartum depression or psychosis. Mother-infant bonding as defined in this study refers to the emotional attachment that normally exist between a mother and her newly born infant. Factors susceptible to impact mother-infant bonding may be maternal, environmental, neonatal, or a combination of all, with possible repercussions on the neonate's neurological and psycho-affective development. Objective: We aimed to identify and assess neonatal determinants of mothers' affective involvement few days after delivery in Cameroonian women. Method: A case-control study was carried out over a period of four months in 2015, in two teaching hospitals of Yaoundé, Cameroon among 321 newly delivered women of which 107 had been diagnosed with emotional instability (the cases), and 214 emotionally stable women (the controls). Results: Neonatal factors associated with mothers' emotional swings also known as the baby blues were babies presenting health problems $(\mathrm{OR}=4.33 ; \mathrm{p}<0.001)$, mothers' difficulties in taking care of the baby $(\mathrm{OR}=7.65 ; \mathrm{p}<0.001)$, having given birth to a baby of female sex $(\mathrm{OR}=1.66 ; \mathrm{p}=0.033)$. Conclusion: Emotional instability and mood swings causing affective impairments in mothers few days after delivery may be associated with a number of neonatal factors which account for its onset. There exists a susceptibility to the alteration of mother-infant bonding which can resound on neonatal neurological and psycho-affective development. This may be prevented by optimal preparation for maternity including baby care practices, perinatal counselling, mothers' assistance, and encouraged breastfeeding.
\end{abstract}

Keywords: Mother-infant Bonding, Emotional Instability, Postpartum, Cameroon

\section{Introduction}

Studies on mother-infant affective attachment over the years have elucidated the postpartum period as a special moment for the establishment of bonding [1]. The strength of this relationship seems to depend on various interaction processes which are based on the emotional and behavioural expressions of both the mother and her baby $[2,3]$. Such emotional involvements, when favourable are believed to improve maternity, promote psycho affective and neurological developments of the infant $[4,5]$. It is therefore a bidirectional process in which the neonate also participates. In effect it has been noted that some neonatal expressions such as crying, eye contact and facial expressions could induce maternal emotional or behavioural responses [6]. Alterations of mother-infant bonding may be multifactorial including maternal factors such as environmental, psychological and hormonal influences during the perinatal period on one side and neonatal factors on the other [7]. Though the psychological aspects of obstetrical care are continuously strengthened through prenatal consultations and counselling systems, about $30 \%$ of newly delivered women may experience emotional instability in our context [8]. 
Some common strange feelings such as indifference, disappointment with regard to the baby contrast with the expected joy, relief or satisfaction [8-10]. Moreover, behavioural impairments such as aggressiveness, depression, anxiety which may occur are not favourable for motherinfant affective attachment. The present study aimed at investigating possible neonatal determinants likely to impact the said relationship.

\section{Methodology}

A case-control study was carried out on an overall sample of 321 newly delivered women out of which 107 had been diagnosed with emotional swings within the ten first days of postpartum and were considered as the group of cases, while the other 214 women who did not manifest emotional instability were considered as the control group. The study was carried out in two teaching hospitals of Yaoundé, Cameroon (the Yaoundé Gynaeco-Obstetric and Paediatric Hospital, and the Yaoundé Central Hospital) which are referral and University teaching hospitals in Cameroon. After approval of the research protocol by the ethical committee, women who delivered at 28 weeks or more of pregnancy were enrolled, after their consent was obtained. A pretested questionnaire was administered and information retrieved from the patients' files. Data collected included sociodemographic characteristics, newborn parameters and the Kennerley and Gath questionnaire. The Kennerley and Gath blues questionnaire is a validated self-rating scale consisting of 28 items concerning the emotional state of newly delivered women. The available answers are "yes" or "no" corresponding respectively to marks of 1 and 0 with a maximum possible score of 28 and a minimum of 0 . The scale served as a diagnostic and explorative tool. Women who had an overall score greater than the mean peak score of their group were considered as positive for emotional instability.

The calculated minimal sample size was 41 cases for 82 controls based on the $31.3 \%$ prevalence of baby blues among Nigerian women in postpartum, reported by Adewuya and using Schlesselman's formula with a standardized power of $84 \%$. Statistical analyses were done using CSPro version 4.1 and SPSS version 22.0 software. The difference between variables assessed was statistically significant for P-value < 0.05. Pearson Chi square and Fisher's exact tests were used to compare proportions. Odds Ratio (OR) and 95\% Confidence interval (CI) were calculated to assess the association between variables and emotional instability. Multivariate analysis served for isolating independent predictive factors.

\section{Results}

Having a baby of female sex was a statistically significant risk factor $(\mathrm{OR}=1.66, \mathrm{p}=0.03)$. Babies with health problems characterized by the notions of absence of afterbirth cry and resuscitation were significantly associated with the onset of mothers' emotional instability $(\mathrm{OR}=4.33, \mathrm{p}<0.001)$. Having given birth to a single baby was not a significant protective factor $(\mathrm{OR}=0.62, \mathrm{p}=0.365)$. These results are summarized in table 1 .

Table 1. Newborns' parameters in relationship with mothers' emotional instability (1).

\begin{tabular}{|c|c|c|c|c|c|}
\hline \multirow{2}{*}{ Newborns' parameters } & \multirow{2}{*}{$\begin{array}{l}\text { Cases }(n=107) \\
\mathrm{n}(\%) \\
\end{array}$} & \multirow{2}{*}{$\begin{array}{l}\text { Controls }(n=214) \\
n(\%) \\
\end{array}$} & \multirow{2}{*}{$\begin{array}{l}\text { Total } \\
\mathbf{N} \\
\end{array}$} & \multirow{2}{*}{ OR [IC 95\%] } & \multirow{2}{*}{$\mathbf{P}$} \\
\hline & & & & & \\
\hline \multicolumn{6}{|l|}{ Number of babies } \\
\hline 1 & $100(93.5)$ & $205(95.8)$ & 305 & $0.63[0.22-1.77]$ & \multirow{2}{*}{0.365} \\
\hline 2 & $7(6.5)$ & $9(4.2)$ & 16 & $1.50[0.59-4.39]$ & \\
\hline \multicolumn{6}{|l|}{ Sex } \\
\hline Female & $63(58.9)$ & $99(46.3)$ & 162 & $1.66[1.04-2.66]$ & \multirow{2}{*}{0.033} \\
\hline Male & $44(41.1)$ & $115(53.7)$ & 159 & {$[0.37-0.96]$} & \\
\hline \multicolumn{6}{|c|}{ Did your baby present health problems? } \\
\hline Yes & $33(30.8)$ & $20(9.3)$ & 53 & $4.33[2.33-8.01]$ & \multirow[t]{2}{*}{$<0.001$} \\
\hline No & $74(69.2)$ & $194(90.7)$ & 268 & $0.23[0.35-0.90]$ & \\
\hline \multicolumn{6}{|l|}{ Baby afterbirth cry? } \\
\hline Yes & $74(69.2)$ & $184(86.0)$ & 258 & $0.37[0.21-0.64]$ & \multirow{2}{*}{$<0.001$} \\
\hline No & $33(30.8)$ & $30(14.0)$ & 63 & $2.70[0.12-0.43]$ & \\
\hline \multicolumn{6}{|l|}{ Baby resuscitation? } \\
\hline Yes & $33(30.8)$ & $33(15.4)$ & 66 & $2.45[1.41-4.25]$ & \multirow{2}{*}{0.001} \\
\hline No & $74(69.2)$ & $181(84.6)$ & 255 & $0.40[0.23-0.70]$ & \\
\hline
\end{tabular}

Mothers' difficulties in taking care of the newborn appeared to be predisposing to emotional instability with statistically significant association $(\mathrm{OR}=7.65$, pvalue $<0.001)$, and so were all its various items evaluated such as difficulties with baby's sleep $(\mathrm{OR}=8.7)$, difficulties in feeding the baby $(\mathrm{OR}=10.52)$, considering the baby irritable (11.7), baby crying a lot $(\mathrm{OR}=9.6)$, difficulties to calm or ease the baby $(\mathrm{OR}=11.92)$. Breastfeeding was a protective factor against mothers' emotional swings $(\mathrm{OR}=0.11$, $\mathrm{p}<0.001)$. These other results as shown in table 2 . 
Table 2. Newborns' parameters in relationship with mothers' emotional instability (2).

\begin{tabular}{|c|c|c|c|c|c|}
\hline \multirow{2}{*}{ Newborns' parameters } & \multirow{2}{*}{$\begin{array}{l}\text { Cases }(n=107) \\
n(\%)\end{array}$} & \multirow{2}{*}{$\begin{array}{l}\text { controls }(n=214) \\
n(\%)\end{array}$} & \multirow{2}{*}{$\begin{array}{l}\text { Total }(n=321) \\
N\end{array}$} & \multirow{2}{*}{ OR [IC 95\%] } & \multirow{2}{*}{$\mathbf{P}$} \\
\hline & & & & & \\
\hline \multicolumn{6}{|c|}{ Do you have difficulties to take care of the baby? } \\
\hline Yes & $12(11.2)$ & $11(5.1)$ & 29 & $7.65[3,15-18,59]$ & \multirow{2}{*}{$<0.001$} \\
\hline No & $95(88.8)$ & $203(94.9)$ & 292 & $0.42[0,18-1.0]$ & \\
\hline \multicolumn{6}{|c|}{ Do you have difficulties with baby's sleep? } \\
\hline Yes & $32(29.9)$ & $10(4.7)$ & 42 & $8.70[4.08-18.57]$ & \multirow{2}{*}{$<0.001$} \\
\hline No & $75(70.1)$ & $204(95.3)$ & 279 & $0.10[0.05-0.25]$ & \\
\hline \multicolumn{6}{|c|}{ Do you have difficulties in feeding the baby? } \\
\hline Yes & $72(67.3)$ & $35(16.4)$ & 107 & $10.52[6.12-18.10]$ & \multirow{2}{*}{$<0.001$} \\
\hline No & $30(28.0)$ & $179(83.6)$ & 214 & $0.08[0.05-0.14]$ & \\
\hline \multicolumn{6}{|c|}{ Do you consider your baby as being irritable? } \\
\hline Yes & $39(36.4)$ & $10(4.7)$ & 49 & 11.70 [5.54-24.69] & \multirow{2}{*}{$<0.001$} \\
\hline No & $68(63.6)$ & $204(95.3)$ & 272 & $0.09[0.05-0.15]$ & \\
\hline \multicolumn{6}{|l|}{ Does your baby cry a lot? } \\
\hline Yes & $43(40.2)$ & $14(13.1)$ & 57 & $9.60[4.93-18.67]$ & \multirow{2}{*}{$<0.001$} \\
\hline No & $64(59.8)$ & $200(95.5)$ & 264 & $0.10[0.05-0.20]$ & \\
\hline \multicolumn{6}{|c|}{ Is your baby difficult to calm / ease? } \\
\hline Yes & $42(39.3)$ & $11(5.1)$ & 53 & $11.92[5.80-24.50]$ & \multirow{2}{*}{$<0.001$} \\
\hline No & $65(60.7)$ & $203(94.9)$ & 268 & $0.08[0.04-0.17]$ & \\
\hline \multicolumn{6}{|l|}{ Breast- feeding? } \\
\hline Yes & $40(37.4)$ & $180(84.1)$ & 220 & $0.11[0.07-0.19]$ & \multirow{2}{*}{$<0.001$} \\
\hline No & 67 (62.6) & $34(15.9)$ & 101 & $8.87[5.16-15.15]$ & \\
\hline
\end{tabular}

After multivariate analysis of various risk factors, mothers' difficulties to take care of the baby appeared to be an independent predictive factor of emotional instability in newly delivered women.

Table 3. Multivariate analysis.

\begin{tabular}{|c|c|c|c|c|c|}
\hline \multirow{2}{*}{$\begin{array}{l}\text { Possible independent predictive factors } \\
\text { for baby blues after caesarean section }\end{array}$} & \multirow{2}{*}{$\begin{array}{l}\text { Cases }(n=107) \\
n(\%) \\
\end{array}$} & \multirow{2}{*}{$\begin{array}{l}\text { Controls }(\mathrm{n}=\mathbf{2 1 4}) \\
\mathrm{n}(\%) \\
\end{array}$} & \multirow{2}{*}{ Coef } & \multirow{2}{*}{ OR [IC 95\%] } & \multirow{2}{*}{$\mathbf{P}$} \\
\hline & & & & & \\
\hline \multicolumn{6}{|l|}{ Difficulties to take care of the baby } \\
\hline Yes & $28(26.2)$ & $16(7.5)$ & 1.222 & $3.40[1.12-10.27]$ & 0.030 \\
\hline
\end{tabular}

\section{Discussion}

A number of neonatal factors significantly associated with mothers' emotional instability were identified in our survey. Having given birth to a baby of female sex as already described by other African researchers tends to be more of a socio-cultural issue in our context, related to the sex of the baby $[5,9,11,12]$. In effect, in some cultures in sub-Saharan Africa, some pregnant women may be subjected to considerable family pressures inherent in tradition and social apprehensions [9]. Priority is often given to a male child, considered as a "potential successor for the father" and "additional labour force" for family activities such as agriculture. Therefore, giving birth to an infant of female sex could be a source of profound deception, psychologically difficult to bear for mothers [9]. This may further complicate integration to maternity, thereby prompting the onset of emotional imbalances.

A baby presenting health problems even though considered as an exclusion criterion in some studies, was found as a risk factor for mothers' emotional upsets in our survey [11-13]. It was investigated under two aspects. The notion of "afterbirth cry" perhaps perceived by mothers as an assurance of baby wellbeing, appeared to be a protective factor while the absence of baby afterbirth cry and the notion of resuscitation were significantly associated with the onset of emotional instability $[14,15]$. This could be explained as contrarily to afterbirth cry, resuscitation seems to bring forth anguish in the mother's mind and may lead to mental fatigue.

Mothers' difficulties to take care of the baby turned out to be the most strongly associated neonatal factor in this study, being an independent predictive factor after multivariate analysis. The various contributory characteristics investigated were: difficulties with baby's sleep, difficulties in feeding the baby, considering the baby irritable, baby crying a lot, difficulties to calm or ease the baby. These findings are in line with Thalassinos' results, given such difficulties may arise as a result of unpreparedness to pregnancy, precocious and unwanted pregnancies and increased pains, reflecting a low level of preparation for maternity and perhaps a weak prenatal counselling system [16]. Moreover, difficulties faced by mothers in taking care of the baby may be anxiogenic, leading to irritability or nervousness in accordance with emotional ambivalence that may characterize pregnant and newly delivered women, as described by Zilboorg [17].

Nevertheless, breastfeeding appeared as a protective factor, as it is believed to calm down the baby, preventing it from crying excessively, thereby reducing stress and 
psychological tension in the breastfeeding mother [18].

\section{Conclusion}

Emotional instability and mood swings causing affective impairments in mothers few days after delivery may be associated with a number of neonatal factors which account for its onset. There exists a susceptibility to the alteration of mother-infant bonding which can resound on neonatal neurological and psycho-affective development. This may be prevented by optimal preparation for maternity including baby care practices, perinatal counselling, mothers' assistance, and encouraged breastfeeding.

\section{Funding}

Private.

\section{Conflict of Interest}

The authors declare that they have o competing interest.

\section{Ethical Approval}

the study was approved by the Institutional Ethics Committee.

\section{Acknowledgements}

Hospitals authorities, all collaborators to this project.

\section{References}

[1] Kennel JH, Trause MA, Klause MH: Evidence for sensitive period in the human mother, in CIBA Symposium: Attachment Edited by Hofer N. New York. Elsevier Publishing Co. 1975.

[2] World Health Organization. WHO recommendations: intrapartum care for a positive childbirth experience. WHO 2018.

[3] Ainsworth MDS: Infant behaviour correlates to maternal attachment, in the competent infant: Research and Commentary. Edited by Stone LJ, Smith HT, Murphey LB. New York, Basic Books. 1974.

[4] Jakin I, Maciejewska M, Fabian Daielewska A, Korabiusz K, Wawrykow A, Stecko M. Emotional disorders in the perinatal period. J Educ Health Sport. 2018; 8: 983-989.

[5] Rezaie-Keikhaie K, Arbabshastan ME, Rafiemanesh H,
Amirshahi M, Mogharabi S, Sarjou AA. Prevalence of the Maternity Blues in the Postpartum Period. J Obstet Gynecol Neoatal Nurs. 2020. https://doi.org/10.1016/j.jogn.2020.01.001.

[6] B. Figueiro, R. Costa, A. Pacheco: Mother-to-Infant Emotional Involvement at Birth. Matern Child Health J. 2009; 13: 539-549.

[7] Zanardo V, Volpe F, de Luca F, Giliberti L, Giustardi A, Parotto $\mathrm{M}$ et al. Maternity blues: A risk factor for anhedonia, anxiety, and depression components of Edinburgh Postnatal Depression Scale. J Matern-Fetal Neonatal Med. 2019; 25: 17.

[8] Shi, P., Ren, H., Li, H., \& Dai, Q. Maternal depression and suicide at immediate prenatal and early postpartum periods and psychosocial risk factors. Psychiatry Research. 2018; 261: 298-306. https://doi.org/10.1016/j.psychres.

[9] Adewuya AO. Prevalence and risk factors of maternity blues in western Nigerian women. Am J OstetGynaecol. 2005; 193 (4): $1522-5$

[10] Mac Farlane JA, Smith DM, Garrow DH: The relationship between mother and neonate. In S. Kitzinger \& JA Davis (Eds). The place of birth. New York, NY: Oxford University Press. 1978.

[11] Robson KS, \& Moss H: Patterns and determinants of maternal attachment. Journal of Pediatrics. 1970; 77: 976985.

[12] Nagata et al. Maternity blues and attachment to children in mothers of full term normal infants. Acta Psychol Scand. 2000; 101 (3): 209-217.

[13] Alexandre FC, Paolo RM. Maternity blues: Prevalence and risk factors. Sp J Psychol. 2008; 2 (11): 593-599.

[14] Fatoye FO, Adeyemi AB, Oladimeji BY. Postpartum depression following normal vaginal delivery among Nigerian women. Psychol Rep. 2004; 94: 1276-1278.

[15] K. Mbaïlara et al. Le baby blues: caracterisation clinique et influence des variables psycho-social. L'encephale. 2005; 31: 331-6.

[16] Thalassinos et al. Anxiety and depressive disorders in the postpartum period in pregnant females. J. Gynecol. Obstet. Biol. Reprod. 1993; 22 (1): 101-106.

[17] Zilboorg G. The dynamic of schizophrenia reactions related to pregnancy and childbirth. Am J Psychiatry. 1929; 85 (20): 733-741.

[18] Fiala1 A, Svancara J, Klanova J, Kasparek T. Sociodemographic and delivery risk factors for developing postpartum depression in a sample of 3233 mothers from the Czech ELSPAC study BMC Psychiatry. 2017; 17: 104. 